\title{
Cohesiveness of Powdered Co-Amorphous Olanzapine and Impact on Tablet Production ${ }^{\dagger}$
}

\author{
Nuno F. da Costa ${ }^{1}{ }^{(}$, João F. Pinto ${ }^{1}\left(\mathbb{D}\right.$ and Ana I. Fernandes ${ }^{2, *}(\mathbb{D}$ \\ 1 iMed.ULisboa, Departamento de Farmácia Galénica e Tecnologia Farmacêutica, Faculdade de Farmácia, \\ Universidade de Lisboa, Av. Prof. Gama Pinto, P-1649-003 Lisboa, Portugal; \\ nparferreira@gmail.com (N.F.d.C.); jfpinto@ff.ul.pt (J.F.P.) \\ 2 CiiEM-Interdisciplinary Research Center Egas Moniz, Instituto Universitário Egas Moniz, \\ Monte de Caparica, P-2829-511 Caparica, Portugal \\ * Correspondence: aifernandes@egasmoniz.edu.pt; Tel.: +351-212-946-823 \\ + Presented at the 5th International Congress of CiiEM-Reducing Inequalities in Health and Society, Online, \\ 16-18 June 2021.
}

check for

updates

Citation: da Costa, N.F.; Pinto, J.F.; Fernandes, A.I. Cohesiveness of Powdered Co-Amorphous Olanzapine and Impact on Tablet Production. Med. Sci. Forum 2021, 5, 2. https://doi.org/2021005002

Academic Editors: Helena Barroso and Cidália Castro

Published: 9 July 2021

Publisher's Note: MDPI stays neutral with regard to jurisdictional claims in published maps and institutional affiliations.

Copyright: (c) 2021 by the authors. Licensee MDPI, Basel, Switzerland. This article is an open access article distributed under the terms and conditions of the Creative Commons Attribution (CC BY) license (https:// creativecommons.org/licenses/by/ $4.0 /)$.

\begin{abstract}
The evaluation of the processability of co-amorphous mixtures is of paramount importance since these systems are increasingly used to address the poor solubility presented by most of the drugs in research and development. This work shows that co-amorphous olanzapine powders present higher cohesiveness than their crystalline counterpart and resulted in the production of tablets with a higher tensile strength and a slower release of the drug. As a result, this work demonstrates that despite the solubility advantages of co-amorphous mixtures, consideration should be given to the downstream processing of formulations containing such materials.
\end{abstract}

Keywords: co-amorphous; cohesiveness; dissolution; olanzapine; tablets; tensile strength

\section{Introduction}

Co-amorphization of drugs has been extensively described as an adequate approach to overcome the poor bioavailability issues faced by a significant fraction of drug substances (approximately 70\%) [1] currently under research and development. Though of paramount importance, assessment of the processability of co-amorphous systems for the manufacture of medicines is still lacking [2]. This work aimed at evaluating the cohesiveness of crystalline vs. co-amorphous olanzapine/saccharin powder mixtures, and the attributes of tablets produced with these materials.

\section{Materials and Methods}

Olanzapine (OLZ, Rampex Labs, Telangana, India) and saccharin (SAC, SigmaAldrich, Steinheim, Germany) were dissolved in dichloromethane (Biochem Chemopharma, Cosne sur Loire, France) and co-amorphized by evaporating the solvent at $45^{\circ} \mathrm{C} / 650 \mathrm{mbar}$ (Buchi Rotavapor, Buchi, Flawil, Switzerland) [3]. Rheological characterization of crystalline physical mixtures of OLZ and SAC and the co-amorphous system was carried out in a TA.XT Plus Texture Analyzer fitted with a powder flow accessory (Stable Micro Systems, Surrey, United Kingdom). The cohesion index was determined as the ratio between the work required to move the blade upwards $(75 \mathrm{~mm} / \mathrm{s})$ and the weight of the sample $(30 \mathrm{~g})$. To produce tablets, OLZ (30\%), either as a crystalline mixture with SAC $(18 \%)$ or in the co-amorphous system, was blended with anhydrous dibasic calcium phosphate $(27 \%$, Budenheim, Budenheim, Germany), microcrystalline cellulose (20\%, FMC Corp., Cork, Ireland) and povidone (5\%, BASF, Ludwigshafen, Germany). Blended samples ( $66.5 \mathrm{mg}$ manually weighted) were used to fill the compression die (5 $\mathrm{mm}$ diameter, Lloyd Instruments, Largo, FL, USA) and compressed (90 MPa at a constant speed of $10 \mathrm{~mm} / \mathrm{min}$ ). Tablets were characterized based on tensile strength (TA.XT Plus fitted with a cylinder 
probe, Stable Micro Systems, Surrey, United Kingdom), disintegration time (demineralized water, $37 \pm 2{ }^{\circ} \mathrm{C}$, Erweka, Heusenstamm, Germany) and dissolution testing using the paddle method ( $100 \mathrm{rpm}, 37 \pm 2{ }^{\circ} \mathrm{C}$, Sotax, Aesch, Switzerland) and $\mathrm{pH} 6.8$ phosphate buffer $(300 \mathrm{~mL})$ as the dissolution medium.

\section{Results and Discussion}

Co-amorphization of OLZ and SAC was previously described as a useful method to enhance the water solubility and dissolution rate of OLZ [3]. However, co-amorphization of OLZ resulted in the production of a powdered system presenting a higher cohesion index $(25.2 \pm 1.2)$ than its crystalline counterpart $(12.7 \pm 0.4)$. The higher cohesiveness of co-amorphous OLZ may be explained by the increased mobility of its molecules at the surface of the particles, thus impacting negatively on the flow. As a consequence of the poor flowability of the co-amorphous OLZ, an unacceptable mass and content uniformity are expected, negatively impacting on the therapeutic effectiveness of the dosage form and rendering batches uncompliant with pharmacopoeia standards.

Tableting of blends containing co-amorphous OLZ resulted in the preparation of compacts with a higher tensile strength $(1.97 \pm 0.01 \mathrm{MPa})$ than those produced with the physical mixture of crystalline OLZ and SAC $(1.01 \pm 0.05 \mathrm{MPa})$. The higher tensile strength of compacts made with a co-amorphous OLZ probably relates to the higher cohesiveness of co-amorphous OLZ, whose particles also seem to be more elastic and plastic than the crystalline equivalents. Moreover, during compaction, the temperature of the powder bed increased and may have approached, or even exceeded, the glass transition temperature of the co-amorphous OLZ $\left(57^{\circ} \mathrm{C}\right)$, favoring the rubbery state of the system. Thus, the disintegration time of tablets made of co-amorphous OLZ was significantly higher $(420.0 \pm 87.2 \mathrm{~s})$ than that of tablets produced with crystalline OLZ/SAC $(48.7 \pm 4.5 \mathrm{~s})$. This difference is also supported by the mechanism of disintegration observed: while for crystalline OLZ/SAC tablets, compacts disintegrated upon contact with water, tablets made of co-amorphous OLZ slowly and continuously eroded. As a consequence, a slower release of OLZ from tablets made of co-amorphous OLZ was particularly relevant at the beginning of the dissolution test (Figure 1) carried out under sink conditions.

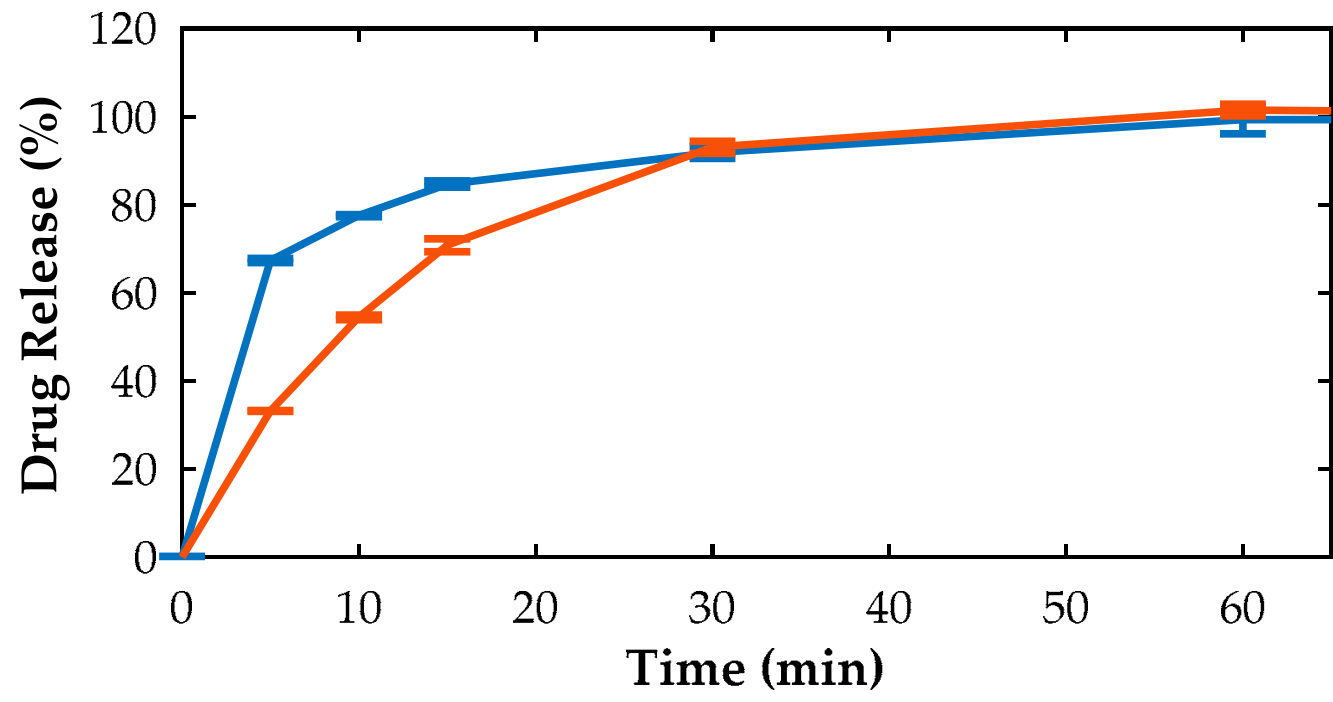

Figure 1. Dissolution profile of tablets produced from physical mixtures of crystalline (blue) and co-amorphous (orange) OLZ/SAC.

In view of the results, downstream processing of formulations containing co-amorphous materials should be given consideration, since they present different bulk flow properties which impact on the quality of the product. 
Informed Consent Statement: Not applicable.

Data Availability Statement: Not applicable.

Acknowledgments: The authors acknowledge Fundação para a Ciência e a Tecnologia, Lisbon, Portugal, for providing financial support to this work (PTDC/CTM-BIO/3946/2014 and SFRH/BD/137080/2018).

Conflicts of Interest: The authors declare no conflict of interest.

\section{References}

1. Da Costa, N.F.; Pinto, J.F.; Fernandes, A.I. Co-amorphization of olanzapine for solubility enhancement. Ann. Med. 2019, 51, 87. [CrossRef]

2. Lenz, E.; Jensen, K.T.; Blaabjerg, L.I.; Knop, K.; Grohganz, H.; Löbmann, K.; Rades, T.; Kleinebudde, P. Solid-state properties and dissolution behaviour of tablets containing co-amorphous indomethacin-arginine. Eur. J. Pharm. Biopharm. 2015, 96, 44-52. [CrossRef] [PubMed]

3. Da Costa, N.F.; Fernandes, A.I.; Pinto, J.F. Measurement of the amorphous fraction of olanzapine incorporated in a co-amorphous formulation. Int. J. Pharm. 2020, 588, 119716. [CrossRef] [PubMed] 